\title{
The new face of Clinical \& Biomedical Research: What REMAINS AND WHAT CHANGES IN 2015
}

\section{Alexandre Prehn Zavascki ${ }^{1,2}$}

The first goal of our journal was achieved in 2014: to change its face. Certain features were added to the more than 30-year-old structure of the journal to make it an international vehicle of dissemination of scientific information in medical and biomedical fields. The publication of an increased number of articles written in English and the considerable participation of external reviewers, including a substantial number of reviewers from foreign institutions ${ }^{1}$, were the first signs that the process was going in the right direction. However, although there is an interesting number of external collaborations, such as articles from foreign authors, our main goal remains to be achieved. In 2015, we aim a higher number of submissions of authors from outside of the institutions responsible for the journal.

The journal was indexed on more databases, most recently on CrossRef, which, in a medium term, will increase its visibility among researchers from other national and international institutions. More publicity for our journal by the responsible institutions, namely School of Medicine of Universidade Federal do Rio Grande do Sul and Hospital de Clínicas de Porto Alegre, also seems to be absolutely necessary to achieve this purpose. Therefore, in this second moment of CBR, we hope to rely on a more solid structure for a systematic divulgation of the journal and the studies published in it.

The core structure of the journal and its new face will not change. The renewed editorial policies will continue, to aim for the studies published in the journal to reach a quality level compatible with that of an international journal. Punctual changes in the editorial board and associate editors sought to cover a more diverse variety of medical and biomedical fields, as well as to maintain and even improve services provided to authors: a serious and fast review process.

In 2015, CBR will begin to receive review articles in Portuguese again. Evaluations after the first year of the journal showed us that there is an important space for good scientific quality reviews in Portuguese. Special articles, original articles, and letters to the editor will continue to be accepted in English, Portuguese, and Spanish. Case reports and Case Reports: Images Medicine will be published only in English. In 2015, we will begin to receive brief communications reporting preliminary or punctual observations (see Instructions for authors).

In editorials published in 2014, we aimed to present an update of all the steps of the new journal. In this year, after the first goals were achieved, we sought to publish editorials with issues relevant to medical and biomedical fields. In general terms, editorials will be published by invitation, but proposals for editorials will be received and analyzed regarding their potential for publication in the journal.

Finally, in a time when there has been considerable discussion about the commercialization of scientific research, we sought to consolidate our position as a free access journal at no cost for authors, following the ethical provisions established by the Committee on Publication Ethics (http://publicationethics. org/) to ensure the integrity of scientific publications from submission through the peer-review process up to the final publication of the article.

Clin Biomed Res. 2015;35(1):3-4

1 Department of Internal Medicine, Medical School, Universidade Federal do Rio Grande do Sul (UFRGS). Porto Alegre, RS, Brazil.

2 Infectious Diseases Service, Hospital de Clínicas de Porto Alegre. Porto Alegre, RS, Brazil.

Corresponding author: Alexandre Prehn Zavascki E-mail: azavascki@hcpa.ufrgs.br Porto Alegre, RS, Brazil. 


\section{REFERENCES}

1. Zavascki AP. Clinical and Biomedical Research: the first year of the journal. Clin Biomed Res. 2014;34(4):331-2. 